
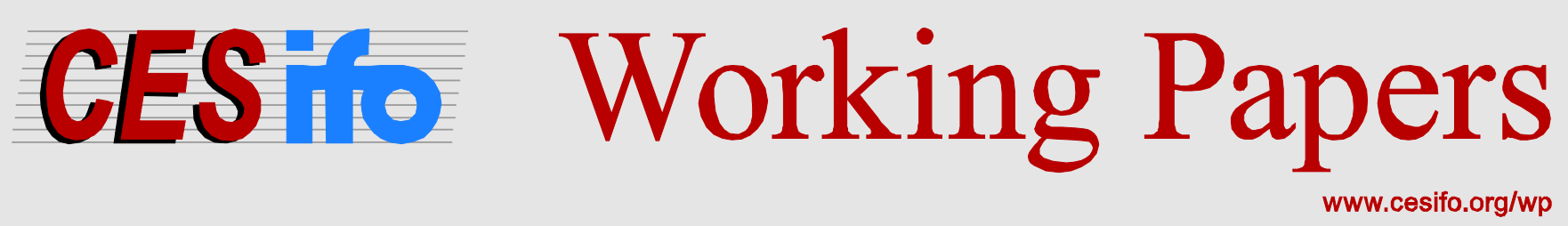

\title{
The Signaling Role of Charitable Contributions by Businesses: A Tax Policy Perspective
}

\author{
Tomer Blumkin \\ Yoram Margalioth \\ Efraim Sadka \\ Adi Sharoni
}

CESIFO WORKING PAPER NO. 6106

CATEgory 1: PubliC FinANCE

SEPTEMBER 2016

An electronic version of the paper may be downloaded

- from the SSRN website:

- from the RePEc website:

- from the CESifo website:

WwW.SSRN.com

www.RePEc.org

www.CESifo-group.org/wp 


\title{
The Signaling Role of Charitable Contributions by Businesses: A Tax Policy Perspective
}

\begin{abstract}
Empirical evidence suggests that charitable contributions to public goods may be driven not only by the familiar warm-glow of giving motive but also as a means for businesses to signal high product quality. Building on this finding, we present an analytical framework that characterizes the optimal government policy, assuming that the government may either directly provide the public good or subsidize its private provision. We show that in the optimal solution the government should subsidize the private provision of the public good and refrain from direct provision. We further demonstrate that the optimal degree of subsidization should decrease with the extent to which the signal is informative, and may even turn into a tax when the signal is sufficiently strong. Finally, we compare the current practice in the US, a charitable contribution deduction provided by Section 170 of the US Tax Code, with the optimal design suggested by our normative analysis and offer changes that would bring the Section closer to the social optimum.
\end{abstract}

JEL-Codes: H200, H400, K300.

Keywords: public goods, Pigouvian taxation, warm glow, signaling.

Tomer Blumkin Department of Economics

Ben-Gurion University

Israel - Beer-Sheba 84105 tomerblu@exchange.bgu.ac.il

Efraim Sadka

The Berglas School of Economics

Tel-Aviv University

Israel - Tel-Aviv 69978

sadka@post.tau.ac.il
Yoram Margalioth

The Buchmann Faculty of Law

Tel-Aviv University

Israel - Tel-Aviv 69978

margalio@post-tau.ac.il

Adi Sharoni

Department of Economics

Ben-Gurion University

Israel - Beer-Sheba 84105

adi.sharoni.n@gmail.com 


\section{Introduction}

Efficient provision of a public good requires that its amount be set to the level that equates the marginal cost of provision and the sum of the marginal benefits derived by all consumers. The private market is unlikely to provide the optimal level of public goods due to several reasons. Foremost among them are: (i) The free-rider problem: public goods are typically non-excludible; hence, individuals are tempted to benefit from them without paying their fair share, or paying at all; (ii) A standard externality problem: selfinterested individuals tend to ignore the benefit derived from the public good by other individuals. This leads to under-provision of public goods and calls for government intervention

Assuming plausibly, in line of (ii) above, that individuals disregard the effect of their contributions on the aggregate level of public good provision, private provision of public good can take place only when people derive utility, known as 'warm glow,' from the act of contribution itself; namely, from being generous towards worthy causes (the sheer joyof-giving). ${ }^{2}$ This may often be the case, but warm-glow does not solve the underprovision problem, as the donors account only for their utility from giving. The utility derived by the beneficiaries is not accounted for. ${ }^{3}$

In light of the above, under-provision of public goods emerges in an unfettered equilibrium. This may be a rationale for the government to subsidize private provision of public goods.

\footnotetext{
${ }^{2}$ See Andreoni $(1989,1990)$. In this paper we focus our analysis on the warm glow effect. Assuming, alternatively, that individuals do account for the impact of their contributions on the aggregate level of public good provision would complicate the analysis without changing the qualitative nature of our results. ${ }^{3}$ Even when donors derive utility from their belief that their contributions increase the donees' utilities, it is nevertheless the utility of the donors, not the donees, that is taken into account.
} 
In this paper, we focus on private provision of public goods by businesses (either incorporated or unincorporated). ${ }^{4}$ We argue that when the donor is a business charitable contributions may still be motivated by the warm glow of the owners, controlling shareholders and top executives, but may also be driven by the desire to maximize profits. To facilitate the exposition, we will henceforth refer to the warm-glow utility derived by the individuals who own or run the firm as the warm-glow derived by the firm.

In addition, businesses may engage in charitable giving to signal high product quality. ${ }^{5}$ The intuition is that businesses that contribute to public goods are perceived by potential customers to be less likely to cheat, say, by reneging on contracts or using materials of lower than promised quality, compared to businesses that do not engage in charitable giving. There is ample empirical support for our claim. The following two studies are especially relevant.

Siegel and Vitaliano (2007) found that businesses that sell experience goods, which are goods one needs to personally experience in order to assess their quality, are more likely to engage in charitable giving than businesses that sell search goods, namely, goods which quality can be easily verified prior to their purchase.

\footnotetext{
${ }^{4}$ In the case of corporations, the private provision of public goods is part of a wider and growing phenomenon referred to as Corporate Social Responsibility (CSR). There is a voluminous literature on CSR. See, e.g., Benabou and Tirole (2010) for a survey of various explanations for the reasons why CSR is growing.

${ }^{5}$ The signaling motive for engaging in charitable contributions has been originally highlighted by Glazer and Konrad (1996) who demonstrate that individuals may choose to donate to the provision of public goods in order to signal about their unobserved income and thereby gaining social status. Glazer and Konrad focus on individuals, whereas the current paper examines the signaling role of charitable contributions by businesses and further examines the tax policy implications.
} 
Elfenbein, Fisman and Mcmanus (2012) used data from a diverse group of eBay sellers and found that consumers respond positively to products tied to charity, particularly when the sellers were relatively new and hence consumers had limited alternative means of assuring quality. Moreover, consistent with charity serving as a quality signal, fewer customer complaints were found among charity-intensive sellers.

In the current study, we present an analytical framework that captures both motives for charitable contributions: warm glow and product-quality signaling. We assume that the quality of the product/service provided by the firm is unobserved by the consumers. We further assume, in line with the empirical evidence reviewed above, the existence of a positive correlation between the propensity of the firm to engage in charitable giving (captured by the derived utility from warm glow) and the quality of its product/service. Finally we assume that private provision of the public good is observable by the consumers. Our assumptions hence imply that firms may choose to use the provision of the public good, not merely due to the warm glow their owners may enjoy, but also in order to signal their product/service quality. We then characterize the optimal government policy, assuming that the government can engage in direct provision of the public good or subsidize its private provision.

We show that due to the presence of a warm-glow motive the optimal policy would be to subsidize the private provision of the public good and refrain from direct provision. We further show that the degree of subsidization rendered to charitable contributions should vary with the correlation between the propensity of the firm to engage in charitable giving and the quality of its product/service, namely the extent to which the signal is informative. 
When the correlation is relatively small, that is, the signal is relatively weak; the provision of the public good would be exclusively driven by warm glow. Firms would then set their contributions at the level that maximizes the warm-glow utility component. In such a case, the level of public good provision would be lower than the socially desirable (efficient) one. A subsidy would hence be called for to internalize the positive externalities and restore efficiency.

In contrast, when the correlation is sufficiently high, namely the signal is relatively strong; the signaling motive would become operative. Firms that derive a utility from warm glow, which, by presumption, are more likely to provide high product-quality, would increase their level of charitable contributions above the level that maximizes their warm-glow utility component. They do so up to the level that deters mimicking by firms that experience no warm glow, which, by presumption, are more likely to provide low product-quality. Namely, firms that derive warm glow make charitable contributions up to the point where firms that derive no warm glow from charitable contributions but engage in charitable giving for strategic reasons only (pretending to provide high-quality product,) are discouraged from doing so.

The incentive to engage in signaling induces firms to increase their level of contribution to the public good, and hence, calls for a lower degree of subsidization provided by the government. It may even result in an excessive level of contributions by firms, exceeding the socially optimal one, which would call for levying a tax on charitable contributions, contrary to conventional wisdom and common practice. 
We further demonstrate that the current practice in the US, a charitable contribution deduction provided by Section 170 of the US tax code, qualitatively mimics the optimal patterns suggested by our normative analysis. When the signaling incentives are moderate, the section effectively provides a subsidy for charitable giving by allowing taxpayers to deduct their charitable contributions from their taxable income even though such contributions are a form of personal consumption due to the warm glow experienced by the donors. As the signaling effect becomes more manifest, inducing higher levels of contribution, Section 170 transforms from offering a subsidy for charitable giving to allowing a full business deduction. ${ }^{6}$ This takes place as firms choose to set their contributions above the level that would maximize their warm glow in order to separate themselves from firms deriving no warm glow. Thus, the marginal dollar spent on charitable contributions is used for signaling, that is, profit maximization purposes, and not driven by the utility from consumption (warm glow). The full deduction of the amount spent on charitable giving, in the presence of signaling, implies that, at the margin, there is neither a subsidy nor a tax on charitable giving. This maintains the tax system neutral, as in the case of any business deduction

Finally, when the signaling effect becomes sufficiently manifest, namely, the levels of contribution are large enough; Section 170 effectively imposes a tax on charitable contributions. This takes place by limiting the deduction to 50 percent of adjusted gross income (AGI) for individuals and 10 percent of AGI in the case of corporations. Limiting

\footnotetext{
${ }^{6}$ We assume that corporations cannot deduct charitable contributions as expenses incurred in the production of income under tax code section 162. Even if it were possible, acknowledging the existence of Section 162 would not have changed the qualitative nature of our results.
} 
the deduction in spite of its being an expense incurred to generate taxable income, is akin to imposing a tax levied on charitable contributions at the margin.

Notice that Section 170 distinguishes between corporations and individuals. Setting a significantly lower threshold for corporations compared to individuals (unincorporated businesses) seems plausible, as corporations engaging in charitable contributions are likely to do so primarily for profit maximization purposes. In contrast, individuals who perform the charitable giving are primarily driven by warm glow. In the case of unincorporated businesses, these are usually the firm's owners, who are also the firm's managers, so there is no conflict of interests (an agency problem). However, in the case of corporations, the individuals that take the decision to engage in charitable giving on behalf of the corporation and who choose the identity of the donees are: the managers, the board of directors or the controlling shareholders (if such exist). This may come at the expense of the non-controlling shareholders whose preferences regarding contribution to charity may differ substantially from those of the controlling shareholders or managers. ${ }^{7}$ It is therefore unlikely that significant corporate charitable giving would take place if it were only the result of managers or controlling shareholders' warm glow. If that were the case, shareholders would sue, arguing there was a breach of corporate fiduciary duties to maximize profits. ${ }^{8}$ Hence, when corporate charitable contributions reach the level of 10 percent of the corporate adjusted gross income, which is significantly lower than the

\footnotetext{
${ }^{7}$ With the rare exception of social enterprises incorporated under recently enacted new legal forms as a low-profit limited liability company (L3C), a benefit corporation, or a flexible purpose corporation.

${ }^{8}$ See, for example, the cases of Ben \& Jerry's and craigslist. The founders of Ben \& Jerry's claimed that corporate law forced them to sell the company to Unilever. As for craigslist, the court ruled in favor of eBay, a minority shareholder in craigslist, which sued Craig Newmark, the founder of craigslist, for using craigslist to further philanthropic goals. The court ruled that "having chosen a for-profit corporate form, the craigslist directors are bound by the fiduciary duties and standards that accompany that form" [Mayer and Ganahl (2014)].
} 
threshold of 50 percent set for individuals' contributions, it is likely to reflect an operative strong signaling motive that gives rise to an excessive provision of the public good and hence warrants imposing a tax on charitable contributions.

Connecting our normative analysis of the socially optimal tax treatment of charitable contributions and the positive analysis of Section 170, we argue that replacing the current system of a fixed rate of deduction supplemented by fixed caps with a system which allows deducting more than a hundred percent of the actual contributions (a multiplier) but is phasing out as the level of contributions increases, would bring the system even closer to the social optimum.

The paper proceeds as follows. Part 2 outlines our model and analyzes the benchmark laissez-faire case, absent of government intervention. In Part 3 we offer a normative analysis, characterizing the socially optimal policy. Part 4 compares the socially optimal policy with the current practice of the tax treatment of charitable contributions by businesses in the US (Section 170 of the US Tax Code). Part 5 suggests a policy reform that would bring the Section closer to the social optimum and offers some concluding remarks.

\section{The Model}

We present a simple setting with just the essential ingredients required to demonstrate our argument. Consider an economy with $M>0$ identical consumers who derive utility from two private goods (denoted $x$ and $y$ ) and a single public good (denoted by $G$ ). For 
tractability we assume a quasi-linear specification for the utility function (shared by all consumers) taking the following form:

(1) $U(x, G, y, \theta)=v(x, \theta)+h(G)+y$,

where $x \in\{0,1\}, \theta$ denotes the quality associated with $x, v(0, \theta)=0, v(1, \theta)=\theta$ and $h$ is increasing, concave and satisfies standard INADA conditions.

Each consumer has, thus, an inelastic demand for one unit of $x$ with an associated reservation price, depending on the quality of the provider, given by $\theta$ (measured in $y$ terms, the price of which is normalized to unity, with no loss in generality). We simplify by plausibly invoking a 'large economy assumption', letting the amount of public good provision, $G$, be a fixed parameter from the point of view of the individual consumer (not depending on his private contribution). This assumption introduces an extreme 'freerider' problem associated with under-provision of the public good, implying zero private contributions of the consumers to the public good in equilibrium. ${ }^{9}$ Finally, we assume that each consumer is endowed with $Y>0$ units of the numeraire good, $y$. Denoting the price of $x$ (as a function of its associated quality $\theta$ ) by $p(\theta)$, the typical consumer's budget constraint is given by:

(2) $y+p(\theta) \cdot x=Y$.

We turn next to the providers of $x$ who are either owners of unincorporated businesses or managers/controlling shareholders of corporations. We assume that there are $N<M$ providers in the market, each producing a single unit of $x$ at zero costs, with no loss in

\footnotetext{
${ }^{9}$ The 'large economy assumption' is tantamount to perfect competition. Relaxing the assumption will not change the gist of our key argument.
} 
generality. $^{10}$

Providers differ in their quality, where we assume that a fraction $0<1-\alpha<1$ of the providers is of low quality [denoted $\theta=\theta^{l}>0$ ], and a complementary fraction of $0<\alpha<1$ of the providers is of high quality [denoted $\theta=\theta^{h}>\theta^{l}$. ${ }^{11} \mathrm{We}$ assume that the quality of the provider is private information, unobserved by the consumers. Consumers are nonetheless assumed to be familiar with the distribution of providers' types. We further assume that providers may affect consumers' valuations (thereby, affecting their ultimate choices) via signaling. In particular, we assume that providers may engage in contributions to the provision of the public good. The provider's level of contribution to the public good is assumed to be observable by the consumers (say, via disclosure in financial statements or other publicly available reports) and may therefore serve as a signaling channel about the quality of the provider. We assume that some of the providers derive a warm-glow utility associated with contributing to the public good [as in Andreoni (1989), (1990)]. These providers are referred to as socially-motivated providers. Specifically, we assume that a fraction $0<q^{h}<1$ of the high-quality providers, and, correspondingly, a fraction $0<q^{l}<q^{h}$ of the low-quality providers, are deriving utility from the 'joy of giving' associated with contributions to the provision of the public good. Our parametric assumptions imply, therefore, the existence of a positive correlation between the propensity of the provider to contribute to public good provision and its associated quality (as perceived by the consumers). The existence of this positive

\footnotetext{
${ }^{10}$ The assumptions on the particular forms of the demand and supply functions are made for tractability and can be relaxed without changing the qualitative nature of our results.

${ }^{11}$ The quality attribute of a firm is associated with its business conduct towards consumers. A high-quality firm is, inter-alia, more likely to abide by contractual obligations (e.g., timely provision of services) and be more attentive to customers' special needs (e.g., available and efficient customer support services).
} 
correlation implies that in the signaling equilibrium (Bayesian) consumers will (correctly) perceive providers exhibiting a higher extent of charitable contributions to be of higher quality (on average). With slight abuse of notation, the parameter $\theta$ in the utility function given in (1) will be replaced by its expected value, conditional on the information available to the consumers (affected by signals conveyed by the providers). The utility of a typical provider is given by the following expression:

$$
R(g, G, y)=\beta \cdot w(g)+h(G)+y,
$$

where $g$ is the extent of contribution to the public good, $h$, as in the consumers' utility specification, captures the utility from public good provision and $w(g)$ captures the warm glow component, where $\beta=1$ for providers that derive warm glow and $\beta=0$ otherwise. Both $h$ and $w$ are assumed increasing, concave and to satisfy INADA conditions. We maintain our 'large economy assumption' and assume that all providers ignore the impact of their contribution on the aggregate level of public good, $G$.

Similar to consumers, providers are assumed to be endowed with $Y>0$ units of the numeraire good, $y$. The typical provider's budget constraint is given by:

$$
y+g=Y+p(g)
$$

where $p(g)$ denotes the price of $x$ charged by the provider, depending on his (observable) level of contribution, $g$. Notice that we normalize the marginal cost of public good provision to unity (measured in units of $y$ ), with no loss of generality.

Finally, assuming that the market for $x$ is competitive, the assumption that $N<M$ combined with the fact that the aggregate demand for $x$ is perfectly elastic (by virtue of 
the reservation property) imply that in equilibrium the price of $x$, for any level of $g$, will coincide with the (common) reservation price of the consumers; that is, consumers' surplus will be fully extracted by the providers. In equilibrium, this reservation price will be equal to the expected quality of the provider conditional on its level of contribution to the public good, $g$. Formally,

$$
p(g) \equiv E(\theta \mid g)
$$

with $E$ denoting the (conditional) expectation operator.

Notice that our modeling assumptions capture the two different motives for providers to engage in charitable giving: a social motive, reflected by the warm-glow component in the utility specification in (3); and, a strategic motive, captured by the term $p(g)$ in the budget constraint given in (4), where, in equilibrium, the price will be shown to be an increasing function of $g$.

We turn next to characterize the equilibrium. As in many other signaling games, two types of equilibria may arise: (i) A separating equilibrium in which only sociallymotivated providers make contributions to the public goods; (ii) A hybrid equilibrium in which both socially-motivated and non-socially-motivated providers make contributions to the public good. However, by applying a standard refinement criterion, invoking the 'Intuitive Criterion' suggested by Cho and Kreps (1987), one can show that a hybridequilibrium is, in fact, unstable (see Appendix A for details). Thus, in the analysis that follows we will confine attention to the (unique) separating equilibrium. 


\subsection{Separating Equilibrium}

In a separating equilibrium providers that derive 'warm glow' are the only ones to contribute to the public good. Rational consumers fully anticipate this and update accordingly their perception about the quality of the provider (conditional on its observed contribution). Let the (common) level of contribution by a typical socially motivated provider be denoted by $\tilde{g}$.

In order to get some idea about the magnitude of $\tilde{g}$, it is useful to imagine what happens in case there is full information about the product quality. In this case only socially motivated providers will contribute to the public good. By virtue of the utility specification in (3), as the price of $x$ [in the budget constraint given in (4)] will be independent of $g$ in such a case, each socially-motivated provider will choose the level of provision that solves the following first-order condition:

(6) $\quad w^{\prime}\left(g^{*}\right)=1$.

Turning back to our model of asymmetric information, it is clear that the level of contribution, $\tilde{g}$, has to be at least as large as $g^{*}$.

Let $\bar{\theta}$ denote the expected quality associated with a typical socially motivated provider (coinciding with the common price charged by all socially motivated providers in equilibrium). Employing Bayes' Rule it follows:

$$
p(\tilde{g})=\bar{\theta}=\frac{\alpha \cdot q^{h} \cdot \theta^{h}+(1-\alpha) \cdot q^{l} \cdot \theta^{l}}{\left[\alpha \cdot q^{h}+(1-\alpha) \cdot q^{l}\right]}
$$

Similarly, denote by $\underline{\theta}$ the expected quality associated with a provider that does not 
exhibit warm glow (coinciding with the common price charged by all such providers in equilibrium). Employing Bayes' Rule it follows:

$$
p(0)=\underline{\theta}=\frac{\alpha \cdot\left(1-q^{h}\right) \cdot \theta^{h}+(1-\alpha) \cdot\left(1-q^{l}\right) \cdot \theta^{l}}{\left[\alpha \cdot\left(1-q^{h}\right)+(1-\alpha) \cdot\left(1-q^{l}\right)\right]} .
$$

It is straightforward to verify that $\bar{\theta}>\underline{\theta}$, by virtue of our assumption that $q^{h}>q^{l}$ and the fact that $\theta^{h}>\theta^{l}$. Note that the term $\bar{\theta}-\underline{\theta}$ measures the pure returns to signaling. This is also the total gain derived by a non-socially-motivated provider from contributing to the public good.

There are two possible scenarios to consider. One possibility is that the latter gain is smaller than $g^{*}$, the lower-bound level of contribution (made by a socially-motivated provider), that is:

(9) $\bar{\theta}-\underline{\theta} \leq g^{*}$.

In such a scenario no signaling will take place in equilibrium. The 'natural' level of provision by socially-motivated providers (namely, $g^{*}$ ) suffices to deter all other (nonsocially-motivated) providers from making contributions to the public good. Clearly, in such a scenario, the aggregate level of public good provision will be the same under symmetric and asymmetric information regimes $-g^{*}$ per socially-motivated provider. The only difference between the two regimes will be in the division of the consumers' surplus across providers. Under symmetric equilibrium high-quality providers (both socially motivated and those exhibiting no warm glow) will charge a price of $\theta^{h}$, whereas all low-quality providers will charge a price of $\theta^{l}$. Under asymmetric information socially motivated providers (both high- and low-quality) will charge a price of $\bar{\theta}$; 
whereas, all other providers that do not exhibit warm glow (both high- and low-quality) will charge a price of $\underline{\theta}$.

A second scenario is one where the inequality in (9) is reversed. In such a case a separating equilibrium will be characterized by the following condition:

(10) $\bar{\theta}-\underline{\theta}=\tilde{g}>g^{*}$.

Namely, a typical socially-motivated provider will increase its level of provision above that level which maximizes the warm-glow component, $g^{*}$, up to the 'entry-deterrence' point, where non-socially-motivated providers are just indifferent between whether to contribute to charity or not (that is, between mimicking socially-motivated providers by contributing $\tilde{g}$ or refraining from contributing altogether).

Clearly, in such a scenario, the aggregate level of public good provision strictly exceeds that obtained under symmetric information.

In the normative analysis that follows we consider these two scenarios ("no-signaling" and "signaling") separately.

\section{A Normative Analysis}

The government is assumed to have three fiscal instruments at its disposal: (i) direct provision of the public good $(G)$; (ii) a tax $(t)$ on charitable contribution (negative, in the case of a subsidy); and (iii) a lump-sum tax (T) levied on both consumers and providers (negative, in case of a transfer). 
An important clarification is in order. The assumption that the government can levy a lump-sum tax implies that regardless of whether the government is choosing to address the under-provision of public goods by direct provision or by subsidizing charity, both can be financed in a distortion-free manner, entailing no deadweight loss. This apparently suggests that the key policy issue at stake; namely, the desirability of rendering preferential tax treatment to charitable giving, is of little relevance; after all, the government can eliminate the market failure without resorting to subsidizing charity. Notice, however, that this conclusion is wrong. In our setting, as will be shown formally below, the preferred way to address the under-provision market failure is by subsidizing charitable giving by socially motivated providers, as these providers derive warm-glow utility gains from contributing to the public good provision. Clearly, in the absence of distortion-free tax instruments, the government would refrain from implementing the first-best subsidy, which fully internalizes the positive externalities, and compromise on a second-best optimum. Nevertheless, relaxing the assumption of availability of a lumpsum tax, which simplifies our analysis, will not change the qualitative nature of our results. $^{12}$

\footnotetext{
${ }^{12}$ In particular, even in the case where taxation entails a deadweight loss, subsidizing charitable contributions would still be preferred to government direct provision of the public good, as long as the price-elasticity of charitable contributions would be sufficiently high. Bakija (2013) surveys the empirical literature on the price-elasticity of charitable contributions and concludes that the elasticity is about unity. In Appendix $\mathrm{C}$ we demonstrate that with unitary elasticity, subsidization is preferred to direct provision even when government expenditure is financed by distortionary taxation.

Subsidizing charitable contributions could be also justified as a means to overcome the asymmetric information problem faced by the government with respect to individuals' preferences over public good provision (an issue we abstract from discussing in the current study). When taxpayers contribute to charitable organizations, they effectively channel tax revenues to their preferred charitable organizations, thereby providing the government with information about their preferences for direct government spending. This information consists not only of the identity of the charitable organization and the preferred public good, but also the intensity of the preference, reflected in the amount they contribute [see the discussion in Levmore (1998)].
} 
We assume that the government is seeking to maximize a utilitarian social welfare function given by the sum of the utilities of both the consumers and the providers: ${ }^{13}$

$$
\begin{aligned}
& W(G, t, T)= \\
& (N+M) \cdot h(G)+N \cdot\left[\alpha \cdot q^{h}+(1-\alpha) \cdot q^{l}\right] \cdot[w[g(t)]-g(t) \cdot(1+t)]- \\
& (N+M) \cdot T+(N+M) \cdot Y+N \cdot\left[\alpha \cdot \theta^{h}+(1-\alpha) \cdot \theta^{l}\right],{ }^{14}
\end{aligned}
$$

where $g(t)$ denotes the level of contribution chosen by a typical socially-motivated provider.

Naturally, the social welfare function is maximized subject to the government revenue constraint:

$$
N \cdot\left[\alpha \cdot q^{h}+(1-\alpha) \cdot q^{l}\right] \cdot[g(t) \cdot(1+t)]+(N+M) \cdot T-G \geq 0 .
$$

There is another constraint, which requires that the government cannot confiscate the contributions and direct them to its general needs (rebating as a lump-sum transfer, in our case). Put differently, the level of public good provision should (weakly) exceed the total amount of contributions:

$$
G-N \cdot\left[\alpha \cdot q^{h}+(1-\alpha) \cdot q^{l}\right] \cdot g(t) \geq 0
$$

\subsection{Separating Equilibrium with No Signaling}

Socially-motivated providers choose their level of contribution so as to maximize the warm-glow component in their utility function, that is, $g(t)$ is given by the implicit solution to $w^{\prime}(g)=1+t$, and satisfies $g(t) \cdot(1+t) \geq \bar{\theta}-\underline{\theta}$.

\footnotetext{
${ }^{13}$ Note that the results hold for any individualistic social welfare function.

${ }^{14}$ Notice that due to the assumptions on the excess demand for $x(N<M)$ and the fact that aggregate demand for $x$ is perfectly elastic, the aggregate (gross) consumers' surplus from $x, \alpha \cdot \theta^{h}+(1-\alpha) \cdot \theta^{l}$, is fully extracted by the firms, which is reflected in the last term of (11) that represents aggregate firms' surplus (recalling our assumption of zero production costs).
} 
The inequality condition ensures that the amount contributed by socially motivated providers would suffice to deter other providers from engaging in charitable giving.

Proposition 1 below characterizes the optimal policy regime in the case where no signaling takes place, that is, when the returns to signaling are sufficiently small $(\bar{\theta}-$ $\left.\underline{\theta} \leq g^{*}\right)$

Proposition 1: The optimal level of the public good is fully financed by a subsidy to charitable contribution (that is, there is no direct government provision of the public good).

Proof: see Appendix B.

The proposition is consistent with the standard result in the literature and the common practice of rendering preferential tax treatment to charitable giving. The subsidy is essentially playing a Pigouvian role in fully internalizing the positive externalities associated with public good provision. The proposition suggests also that the underprovision of the public good is exclusively addressed by the tax incentives given to socially-motivated providers, and not supplemented by direct provision of public good by the government itself. The reason for the result follows from the warm-glow component in the utility of socially-motivated providers, which makes it more efficient to employ government revenues in order to induce them to increase their private contributions than to provide directly the public good.

\section{2 Separating Equilibrium with Signaling}


In this case, $g(t)$, the level of contribution chosen by a typical socially-motivated provider, is given by the implicit solution to $g(t) \cdot(1+t)=\bar{\theta}-\underline{\theta} \cdot{ }^{14}$

Proposition 2 characterizes the optimal policy regime in the case where signaling does take place, that is, when the returns to signaling are sufficiently large $\left(\bar{\theta}-\underline{\theta}>g^{*}\right)$.

Proposition 2: When the price elasticity of charitable contributions is sufficiently large: (i) there should be no direct provision of the public good by the government; (ii) there exists a unique threshold of the returns on signaling, $(\bar{\theta}-\underline{\theta})$, above which the government levies a tax on charitable contributions, and below which the government subsidizes contributions, albeit at a lower rate than in the no-signaling case; (iii) the tax (subsidy, if negative) on contributions is increasing (decreasing, in the case of a subsidy) with respect to the returns on signaling.

\section{Proof: see Appendix D.}

We turn next to interpret proposition 2. As in the previous case with no signaling, the government should refrain from direct provision of the public good, due to the presence of warm glow. Unlike the previous scenario in which the optimal solution unambiguously suggests that the government should subsidize charitable contributions, in the current scenario, whether the government should tax or subsidize contributions depends on the magnitudes of two conflicting effects: (i) a pigouvian motive to internalize the positive externalities associated with the provision of the public good, calling for a subsidy; (ii) a corrective motive to mitigate the extent of wasteful contributions driven by pure-

\footnotetext{
${ }^{14}$ Thus, signaling does take place in equilibrium and contributions are set to the level which renders the non-socially motivated providers just indifferent between contributing or not, $g(t) \cdot(1+t)=\bar{\theta}-\underline{\theta}$. For the signaling motive to be operative the amount of contributions obtained in equilibrium should exceed the level, which maximizes the warm glow component in the utility of socially motivated providers. Formally, denoting by $\hat{g}(t)$ the implicit solution to $w^{\prime}(g)=1+t$, the following condition has to hold: $\hat{g}(t) \cdot(1+t)<\bar{\theta}-\underline{\theta}$. The latter inequality condition will hold when the returns on signaling, captured by the term $\bar{\theta}-\underline{\theta}$, are sufficiently large (see Appendix D for details).
} 
signaling, justifying a tax. The balance between these two effects depends on the extent of the returns on signaling, captured by the term $\bar{\theta}-\underline{\theta}$. When the returns on signaling are sufficiently low, the pigouvian motive prevails, suggesting the provision of a subsidy (albeit lower than the optimal subsidy provided under the regime with no signaling in place). As the returns on signaling increase, the second effect becomes more manifest, suggesting a decrease in the level of the subsidy provided to charitable contributions. When the returns to signaling are sufficiently large, the market equilibrium results in an excessive level of contributions, reflected in the fact that socially motivated providers set their contributions at a level where the marginal utility derived from an additional dollar contributed to the public good is lower than that associated with an additional dollar spent on the consumption of $y$. As the government, by assumption, cannot directly confiscate the private contributions, the optimal response would be to levy a tax on contributions. ${ }^{15}$ By taxing contributions and rebating the tax-revenues as a lump sum transfer the government diverts resources from contributions (to the public good) to consumption (of $y)$, and thereby enhances welfare.

\footnotetext{
${ }^{15}$ The desirability of taxing charitable contributions is in contrast to conventional wisdom and the common practice of rendering a favorable tax treatment to donations. The result is similar in spirit to Blumkin and Sadka (2007) that focus on individual donations and show that, in the presence of status signaling concerns, the optimal tax on charitable contributions would be non-negative.
} 


\subsection{Summary}

The following illustrative figure depicts the optimal level of tax levied on charitable contributions, $t$, as a function of the returns on signaling, captured by the term $\bar{\theta}-\underline{\theta}$ :

Figure 1 about here

The flat portion represents a regime in which setting charitable contribution at the level that maximizes the warm-glow component suffices to 'deter entry' of non-socially motivated providers. The optimal tax is negative (a subsidy) and fully internalizes the positive externalities associated with the provision of the public good. The rising portion represents a regime in which charitable contributions exceed the level that maximizes the warm-glow component, and hence serve for signaling purposes. With an operative signaling motive in place, the optimal subsidy decreases as the magnitude of the returns on signaling increases. When the returns on signaling become sufficiently manifest, the optimal subsidy on charitable giving turns into a tax, reflecting a scenario in which the public good is over-provided.

\section{Implementation}

In the previous section we have provided a full characterization of the social optimum and demonstrated the relationship between the optimal tax on charitable contributions and the degree of asymmetric information between providers and consumers, captured by the term $\bar{\theta}-\underline{\theta}$, which measures the magnitude of the returns on signaling. In the current section we turn to compare the current practice in the US with the social optimum 
characterized in the previous section by closely examining the policy implications of the deduction on charitable contributions provided by Section 170 under the current US Tax Code.

We let $0<s<1$ denote a proportional income tax (either personal income tax or corporate tax). We turn first to examine the equilibrium configuration in which no signaling takes place and then analyze the case with an operative signaling motive.

\subsection{Equilibrium with No Signaling}

Assuming that the level of contributions is lower than the cap stipulated by Section 170, so that the entire amount contributed is fully tax-deductible, we obtain the following modified first-order condition [compare with the laissez-faire first-order condition given in (6)]:

(14) $w^{\prime}(g)=1-s$.

The deduction of charitable contributions is thus equivalent to a subsidy provided to charitable contributions at a rate equal to the proportional income tax, $s$.

Our presumption that no signaling takes place would be consistent with the definition of the separating equilibrium when the following condition holds:

$$
g(s) \geq \bar{\theta}-\underline{\theta},
$$

where $g(s)$ is given by the implicit solution to (14). As the the term on the left-hand-side of (15) is independent of $\bar{\theta}-\underline{\theta}$, it follows that when the returns on signaling are sufficiently small the deduction policy is indeed equivalent to a subsidy rendered to charitable contributions. The latter serves to internalize the positive externalities associated with the provision of the public good, qualitatively mimicking the pattern 
suggested by our normative analysis. Notice, however, that only in the (knife-edge) case in which $s=-t^{*}$, where $t^{*}$ denotes the socially optimal subsidy (see the derivation in Appendix B), the deduction precisely implements the first-best allocation (under the regime with no signaling in place). ${ }^{16}$

\subsection{Equilibrium with Signaling}

We turn now to the case with an operative signaling motive, namely, a regime in which the condition in (15) is violated. Let the cap stated by Section 170 be denoted by $\bar{g}>$ $g(s)$, where $g(s)$ is given by the implicit solution to (14). Under the regime with signaling in place the amount of charitable contributions, $g$, is set at the level, which renders non-socially motivated providers just indifferent between contributing and refraining from doing so. Formally:

$$
(1-s)[\bar{\theta}-\min (g, \bar{g})]-[g-\min (g, \bar{g})]=(1-s) \underline{\theta} .
$$

We turn next to derive the effective marginal tax rate levied on charitable contributions as a function of the returns on signaling, $\bar{\theta}-\underline{\theta}$. The effective marginal tax rate, $\tau$, is given by the implicit solution to:

$$
\bar{\theta}-\underline{\theta}=g(1+\tau)
$$

\footnotetext{
${ }^{16}$ Notice the subtle difference between condition (15) and the parallel consistency condition [(B7)] in the normative analysis. Condition (B7) requires that the total amount of contribution net of taxes would exceed the term $\bar{\theta}-\underline{\theta}$, in order to induce no mimicking on behalf of non-socially motivated providers (thereby incentivizing them to refrain from engaging in chartable giving). In contrast, condition (15) requires that the total amount of contributions gross of taxes would exceed the term $\bar{\theta}-\underline{\theta}$. The reason for the difference derives from the fact that non-socially motivated providers exhibit no warm-glow from charity and, hence, a deduction of contributions is in their case equivalent to a full deduction of a business expense, which does not affect the choice of the non-socially motivated provider (between engaging in charitable giving or refraining from doing so). Condition (15) qualitatively mimics, hence, condition (9), which guarantees the existence of a separating equilibrium with no signaling in the absence of government intervention.
} 
where $g$ is given by the implicit solution to (16). The condition states that with a tax on contributions, $\tau$, in place, non-socially motivated providers are indifferent between contributing $g$ and not contributing altogether.

We need to separate between two different cases. Consider first the case in which $g(s)<\bar{\theta}-\underline{\theta}<\bar{g}$. In such a case, the solution to (16) is obtained for $g<\bar{g}$, namely, the amount of contributions is lower than the cap and is therefore entirely deductible. Rearranging the expression in (16) then yields:

$$
g=\bar{\theta}-\underline{\theta}
$$

It therefore follows from (17) that the effective marginal tax rate is zero $(\tau=0)$.

Turning next to the case in which $\bar{\theta}-\underline{\theta}>\bar{g}$, the solution to (16) is obtained for $g>\bar{g}$, namely, the cap is binding and only a fraction of the amount of charitable contributions is deductible. Re-arranging the expression in (16) then yields:

$$
(1-s)(\bar{\theta}-\underline{\theta})+s \bar{g}=g<\bar{\theta}-\underline{\theta}
$$

where the last inequality follows as $\bar{\theta}-\underline{\theta}>\bar{g}$.

It therefore follows from (17) that the effective marginal tax rate is positive $(\tau>0)$. Moreover, substituting for $g$ from (19) into (17) yields:

(20) $1+\tau=\frac{\bar{\theta}-\underline{\theta}}{(1-s)(\bar{\theta}-\underline{\theta})+s \bar{g}}$.

It is straightforward to observe that the expression on the right-hand side of (20) is increasing with respect to the term $\bar{\theta}-\underline{\theta}$. 


\subsection{Summary}

The deduction provided by Section 170, supplemented by a cap setting an upper bound on the amount of contributions that are tax deductible, maintain the qualitative patterns suggested by our normative analysis. The subsidy provided to contributions should be decreased when the returns on signaling are sufficiently large, eventually turning into a tax that increases with the returns on signaling.

The following illustrative figure summarizes our positive analysis by depicting the effective marginal tax levied on charitable contributions, $\tau$, associated with the deduction provided by Section 170, as a function of the returns on signaling, captured by the term $\bar{\theta}-\underline{\theta}:$

Figure 2 about here

The flat portion represents a regime in which setting charitable contribution at the level that maximizes the warm-glow component suffices to 'deter entry' of non-socially motivated providers. The effective marginal tax is negative (a subsidy) and equal (in absolute terms) to the marginal income tax rate, $s$. When $\bar{\theta}-\underline{\theta}=g(s)$, the signaling motive becomes operative and the effective marginal tax jumps discontinuously to zero and remains constant until the amount of contributions reach the cap level, $\bar{g}$. The rising portion represents a regime in which only a fraction of the amount of charitable contributions is tax deductible. In this range the effective marginal tax rate is positive and is rising with respect to the term $\bar{\theta}-\underline{\theta}$. 


\section{Suggested Policy Reform and Concluding Remarks}

Comparing figures 1 and 2 that represent, correspondingly, the normative (social optimum) analysis and the positive implication of the current practice of providing a deduction on charitable contributions, calls for an efficiency-enhancing policy reform. The government may supplement the deduction provided by Section 170 with a charitable contributions tax credit exhibiting a phase out regime over an intermediate range of contribution levels, $\overline{\bar{g}}<g<\bar{g}$. This extra tax incentive for charity (gradually diminishing as the returns on signaling increase) would smooth the discontinuous jump in the marginal tax rate associated with the current practice and mimic the patterns suggested by our normative analysis for the intermediate range of charitable contributions.

A key implication of our analysis of current practice under the US Tax Code is two-fold. First, the current practice of providing a deduction commonly perceived as a means to promote charity, may in fact be working in the opposite direction (by levying an effective marginal tax on contributions). The latter may still be warranted, in line with our normative analysis, when signaling incentives are sufficiently manifest. Second, when subsidizing charitable activity is warranted on normative grounds, providing a full deduction of contributions may not suffice and additional tax incentives in the form of (phased-out) tax credits given to charitable contributions may be justified.

From a normative perspective, a key policy implication is the superiority of rendering a preferential tax treatment to charitable contributions by businesses to direct provision of public goods by the government, as a means to address the under-provision market failure associated with privately provided public goods. 


\section{Appendix A: Hybrid-Equilibrium}

The purpose of this appendix is two fold: (i) to characterize the hybrid-equilibrium of the charitable contributions game; (ii) to demonstrate that any hybrid-equilibrium is unstable.

In a hybrid-equilibrium all socially motivated providers as well as a fraction of the nonsocially motivated providers will contribute to the public good, and will choose the same level of contribution. ${ }^{17}$ Let the (common) level of contribution be denoted by $\hat{g}$ and let the fraction of contributing non-socially motivated firms be denoted by $0<\mu<1$. $^{18}$ The complementary fraction of non-socially motivated firms will refrain from contributing, by virtue of our 'large economy assumption'. In equilibrium, each non-socially motivated provider has to be just indifferent between contributing $\hat{g}$ and not contributing at all. ${ }^{19}$

Let $\overline{\bar{\theta}}$ denote the expected quality associated with providers that engage in charitable contributions (coinciding with the common price charged by these providers in equilibrium). Employing Bayes’ Rule it follows:

(A1) $p(\hat{g})=\overline{\bar{\theta}}=\frac{\alpha \cdot q^{h} \cdot \theta^{h}+(1-\alpha) \cdot q^{l} \cdot \theta^{l}+\mu \cdot\left[\alpha \cdot\left(1-q^{h}\right) \cdot \theta^{h}+(1-\alpha) \cdot\left(1-q^{l}\right) \cdot \theta^{l}\right]}{\alpha \cdot q^{h}+(1-\alpha) \cdot q^{l}+\mu \cdot\left[\alpha \cdot\left(1-q^{h}\right)+(1-\alpha) \cdot\left(1-q^{l}\right)\right]}$.

Similarly, denote by $\underline{\underline{\theta}}$ the expected quality associated with providers that do no engage in charitable contributions (coinciding with the common price charged by these providers in equilibrium). Employing Bayes' Rule it follows:

\footnotetext{
${ }^{17}$ A pooling equilibrium in which all firms contribute to the provision of the public good (and choose the same level of contribution) is obtained as a limiting case of the hybrid equilibrium configuration.

${ }^{18}$ As, from a consumer's point of view, high- and low-quality firms choosing the same level of contribution are indistinguishable, we naturally assume that the same fraction of high- and low-quality non-socially motivated firms engages in charitable giving.

${ }^{19}$ In a pooling equilibrium each non-socially motivated firm will weakly prefer to contribute $\hat{g}$ to not contributing at all.
} 
(A2) $\quad p(0)=\underline{\underline{\theta}}=\frac{\alpha \cdot\left(1-q^{h}\right) \cdot \theta^{h}+(1-\alpha) \cdot\left(1-q^{l}\right) \cdot \theta^{l}}{\left[\alpha \cdot\left(1-q^{h}\right)+(1-\alpha) \cdot\left(1-q^{l}\right)\right]}=\underline{\theta}$,

where the last equality follows from (8).

Employing the condition in (7) one can show that:

(A3) $\overline{\bar{\theta}}=\frac{\bar{\delta} \cdot \bar{\theta}+\mu \cdot \underline{\delta} \cdot \underline{\theta}}{\bar{\delta}+\mu \cdot \underline{\delta}}>\underline{\underline{\theta}}$

with $\bar{\delta}=\left[\alpha \cdot q^{h}+(1-\alpha) \cdot q^{l}\right], \underline{\delta}=\left[\alpha \cdot\left(1-q^{h}\right)+(1-\alpha) \cdot\left(1-q^{l}\right)\right]$, and where the inequality sign follows from (A2) and the fact that $\bar{\theta}>\underline{\theta}$.

In a hybrid-equilibrium the common level of contribution of providers that engage in charitable contributions, $\hat{g}$, has to satisfy:

(A4) $\overline{\bar{\theta}}-\underline{\underline{\theta}}=\hat{g}$.

Namely, non-socially motivated providers are just indifferent between contributing $\hat{g}$ and not contributing at all.

Re-arranging (A4), employing (A2) and (A3), yields, following some algebraic manipulations:

(A5) $\mu=\frac{\bar{\delta} \cdot(\bar{\theta}-\underline{\theta}-\hat{g})}{\underline{\delta} \cdot \hat{g}}$.

A hybrid-equilibrium exists if-and-only-if $0<\mu<1$. In addition, as in the case of separating equilibrium, $\hat{g}>g^{*}$; namely, the common level of contribution has to exceed that level which maximizes the utility from warm-glow [defined implicitly by condition 
(6)]. Combining these two conditions, employing (A5), implies that a hybrid-equilibrium exists if-and-only-if:

(A6) $\max \left[\frac{\bar{\delta} \cdot(\bar{\theta}-\underline{\theta})}{\bar{\delta}+\underline{\delta}}, g^{*}\right]<\hat{g}<\bar{\theta}-\underline{\theta}$.

It is straightforward to verify that a hybrid-equilibrium exists if-and-only-if a separating equilibrium with signaling exists; namely, $\bar{\theta}-\underline{\theta}>g^{*}$.

We turn next to show that any hybrid equilibrium is unstable. In particular we will show that a socially motivated provider would gain by deviating from the common level of provision, $\hat{g}$, to a higher level of contribution. In contrast, non-socially motivated providers will find mimicking (adopting a similar deviation strategy) unprofitable.

Consider a deviation from the common level of provision, $\hat{g}$, to some higher level of contribution $g^{\prime}, \hat{g}<g^{\prime} \leq \bar{\theta}$, recalling that $\bar{\theta}$ denotes the expected quality of a socially motivated provider in a separating equilibrium, given by condition (7). We will show that such a deviation exists where it will only be profitable for socially motivated providers to deviate. Consumers will then correctly perceive this to be the case; hence, the price charged by a deviating socially motivated provider will be given by $\bar{\theta}$.

A deviation, $g^{\prime}$, fulfilling the above conditions has to satisfy the following two strict inequalities:

(A7) $\bar{\theta}-g^{\prime}<\overline{\bar{\theta}}-\hat{g}$,

(A8) $\bar{\theta}+w\left(g^{\prime}\right)-g^{\prime}>\overline{\bar{\theta}}+w(\hat{g})-\hat{g}$. 
Condition (A7) states that a non-socially motivated provider finds it strictly unprofitable to deviate; whereas, condition (A8) states that a socially motivated provider finds it strictly profitable to deviate.

We need to show that there exists such $g^{\prime}$ that satisfies both (A7) and (A8). There are two scenarios to consider. Suppose, first, that the inequality condition given in (A8) holds for $g^{\prime}=\bar{\theta}$. Substituting for $g^{\prime}$ into condition (A7) yields:

(A9) $\overline{\bar{\theta}}-\hat{g}>0$,

where the inequality sign in (A9) holds by virtue of condition (A4), which implies that $\overline{\bar{\theta}}-\underline{\underline{\theta}}=\hat{g}$, and the fact that $\underline{\underline{\theta}}>0$.

In such a scenario, $g^{\prime}=\bar{\theta}$ satisfies both (A7) and (A8) as required.

Suppose, alternatively, that for $g^{\prime}=\bar{\theta}$ the inequality condition in (A8) is reversed. Formally, substituting for $g^{\prime}$ into (A8), presuming the inequality sign is reversed, yields:

$$
w(\bar{\theta}) \leq \overline{\bar{\theta}}+w(\hat{g})-\hat{g} .
$$

There are two possibilities to consider. Suppose first that the condition in (A10) is satisfied as equality; namely,

$$
w(\bar{\theta})=\overline{\bar{\theta}}+w(\hat{g})-\hat{g} .
$$

Clearly, by virtue of (A9), the inequality condition in (A7) holds for $g^{\prime}=\bar{\theta}$. Then, by continuity considerations, one can set the level of contribution to be slightly lower than 
$\bar{\theta}$; namely, $g^{\prime \prime}=\bar{\theta}-\varepsilon$, where $\varepsilon>0$ and small, and still maintain the inequality condition in (A7). Furthermore, it follows that:

$$
\bar{\theta}+w\left(g^{\prime \prime}\right)-g^{\prime \prime}>w(\bar{\theta})=\overline{\bar{\theta}}+w(\hat{g})-\hat{g}
$$

where the inequality in (A12) follows as $g^{*}<g^{\prime \prime}<\bar{\theta}$ and by virtue of the fact that the term $\left[w(g)\right.$-g] is decreasing in $g$ for $g>g^{*}$, recalling the concavity of $w$ and the fact that $w^{\prime}\left(g^{*}\right)=1$. Thus, both conditions (A7) and (A8) are satisfied for $g^{\prime \prime}$.

Finally, suppose that the condition in (A10) is satisfied as a strictly inequality. Formally,

$$
w(\bar{\theta})<\overline{\bar{\theta}}+w(\hat{g})-\hat{g} .
$$

Substituting for $g^{\prime}=\hat{g}$ into condition (A8) yields:

$$
\bar{\theta}>\overline{\bar{\theta}}
$$

which holds by virtue of (A3) and the fact that $\bar{\theta}>\underline{\theta}$.

By the continuity of $w$, invoking the intermediate-value theorem, conditions (A13) and (A14) imply that there exist some level of contribution $g^{\prime}, \hat{g}<g^{\prime}<\bar{\theta}$, for which:

$$
\bar{\theta}+w\left(g^{\prime}\right)-g^{\prime}=\overline{\bar{\theta}}+w(\hat{g})-\hat{g}
$$

As $w$ is increasing and $\hat{g}<g^{\prime}$, the condition in (A15) implies:

(A16) $\bar{\theta}-g^{\prime}<\overline{\bar{\theta}}-\hat{g}$.

Thus, the inequality condition in (A7) holds for $g^{\prime}$ given by the implicit solution to (A15). By continuity considerations, one can set the level of contribution to be slightly 
lower than $g^{\prime}$; namely, $g^{\prime \prime}=g^{\prime}-\varepsilon$, where $\varepsilon>0$ and small, and still maintain the inequality condition in (A7). Furthermore, it follows that:

(A17) $\bar{\theta}+w\left(g^{\prime \prime}\right)-g^{\prime \prime}>\bar{\theta}+w\left(g^{\prime}\right)-g^{\prime}=\overline{\bar{\theta}}+w(\hat{g})-\hat{g}$,

where the inequality in (A17) follows as $g^{*}<g^{\prime \prime}<g^{\prime}$ and by virtue of the fact that the term $[w(g)-g]$ is decreasing in $g$ for $g>g^{*}$, recalling the concavity of $w$ and the fact that $w^{\prime}\left(g^{*}\right)=1$. Thus, both conditions (A7) and (A8) are satisfied for $g^{\prime \prime}$. This concludes the proof. 


\section{Appendix B: Proof of Proposition 1}

The Lagrangean of the government optimization problem is given by:

$$
\begin{aligned}
L= & W(G, t, T)+\lambda_{1} \cdot\left[N \cdot\left[\alpha \cdot q^{h}+(1-\alpha) \cdot q^{l}\right] \cdot[g(t) \cdot(1+t)]+(N+M) \cdot T-G\right] \\
& +\lambda_{2} \cdot\left[G-N \cdot\left[\alpha \cdot q^{h}+(1-\alpha) \cdot q^{l}\right] \cdot g(t)\right],
\end{aligned}
$$

with $\lambda_{i}, i=1,2$, denoting, respectively, the Lagrange multipliers associated with the revenue constraint in (12) and the public good provision constraint in (13).

Formulating the first-order conditions with respect to $G, t$ and $T$, yields, correspondingly:

$$
\begin{aligned}
& \frac{\partial L}{\partial G}=(N+M) \cdot h^{\prime}(G)-\lambda_{1}+\lambda_{2}=0, \\
& \frac{\partial L}{\partial t}=N \cdot\left[\alpha \cdot q^{h}+(1-\alpha) \cdot q^{l}\right] \cdot\left[w^{\prime}[g(t)] \cdot g^{\prime}(t)-g^{\prime}(t) \cdot(1+t)-g(t)\right] \\
& +\lambda_{1} \cdot N \cdot\left[\alpha \cdot q^{h}+(1-\alpha) \cdot q^{l}\right] \cdot\left[g^{\prime}(t) \cdot(1+t)+g(t)\right] \\
& -\lambda_{2} \cdot N \cdot\left[\alpha \cdot q^{h}+(1-\alpha) \cdot q^{l}\right] \cdot g^{\prime}(t)=0, \\
& \text { (B3) } \frac{\partial L}{\partial T}=(N+M) \cdot\left(\lambda_{1}-1\right)=0 \leftrightarrow \lambda_{1}=1 \text {. }
\end{aligned}
$$

Substituting for $\lambda_{1}$ and $\lambda_{2}$ from (B3) and (B1) and the individual first-order condition, $w^{\prime}(g)=1+t$, into (B2), yields upon re-arrangement:

$$
\frac{\partial L}{\partial t}=N \cdot\left[\alpha \cdot q^{h}+(1-\alpha) \cdot q^{l}\right] \cdot\left[(N+M) \cdot h^{\prime}(G)+t\right] \cdot g^{\prime}(t)=0
$$

As $g^{\prime}(t)<0,{ }^{20}$ it follows that:

$$
t=-(N+M) \cdot h^{\prime}(G)
$$

Notice that the (absolute value of the) term on the right-hand-side of (B5) captures the marginal social-benefit (spillover) derived from provision of an additional unit of the public good. Thus, condition (B5) implies that the tax on charitable contributions is

\footnotetext{
${ }^{20}$ Full differentiation of the individual first-order condition, $w^{\prime}(g)=1+t$, with respect to $t$, yields, $g^{\prime}(t)=\frac{1}{w^{\prime \prime}[g(t)]}<0$, where the inequality sign follows from the concavity of $w$.
} 
negative (a subsidy) and fully internalizes the positive externalities associated with the public good provision.

We turn next to show that the government refrains from direct provision of the public good. To do so it suffices to show that the constraint given in condition (13) is binding in the optimal solution. To see this, suppose by negation that the constraint in (13) is not binding; hence, $\lambda_{2}=0$. Substituting into the first order condition in (B2), employing the individual first-order condition, yields upon re-arrangement:

$$
\frac{\partial L}{\partial t}=N \cdot\left[\alpha \cdot q^{h}+(1-\alpha) \cdot q^{l}\right] \cdot\left[g^{\prime}(t) \cdot(1+t)\right]<0,
$$

where the inequality sign follows as $g^{\prime}(t)<0$.

We thus obtain a contradiction to optimality [violating the first-order condition in (B2)]. Our final step is to verify that, when the returns on signaling are sufficiently low, our assumption that providers set their contribution at the level that maximizes the warmglow component in their utility is consistent with the optimal solution for the government problem. Substituting for $G$, the aggregate level of public good provision, from condition (13) satisfied as an equality into condition (B5), let $t^{*}$ denote the optimal subsidy given by the implicit solution to (B5), where $g\left(t^{*}\right)$ is given by the implicit solution to the individual first-order condition $w^{\prime}(g)=1+t^{*}$. It follows that the solution is consistent if-and-only-if the following condition holds:

$$
g\left(t^{*}\right) \cdot\left(1+t^{*}\right) \geq \bar{\theta}-\underline{\theta}
$$

Condition (B7) implies that the level of contributions that maximizes the warm-glow component in the utility of socially motivated providers (given the optimal subsidy, $t^{*}$ ) suffices to deter mimicking by non-socially motivated providers. Notice that the expression on the left-hand side of (B7) is independent of $\bar{\theta}-\underline{\theta}$. Thus, when the returns 
on signaling, as captured by the term $\bar{\theta}-\underline{\theta}$, are sufficiently low, the condition given in (B7) is satisfied. This concludes the proof. 


\section{Appendix C: The Dominance of Subsidization over Direct Provision in the Presence of Distortionary Taxation}

In our normative analysis we assume that a lump-sum tax is available to the government and demonstrate that in the social optimum the under-provision of the public good is exclusively addressed by subsidization of socially motivated providers, and not supplemented by direct provision of public good by the government itself. The reason for the result follows from the warm-glow component in the utility of socially motivated providers. In this appendix we extend this result to the case where government expenditure is financed by distortionary taxation.

Notice that although direct provision, by construction, results in no welfare gains from warm glow, it may still be preferred to subsidization when the government can reduce its overall expenditure level by shifting from subsidization to direct provision, thereby mitigating the entailed deadweight loss. If the resulting reduction in the deadweight loss is sufficiently large it will more than offset the forgone gains associated with warm glow. In the presence of distortionary taxation, a sufficient condition for subsidization to dominate direct provision is, therefore, that the government expenditure under a subsidization regime would not exceed the expenditure associated with direct provision, where both regimes yield the same level of public good provision.

Formally, letting $g(1-s)$ denote the demand for charitable contributions by a typical socially motivated provider as a function of the (net-of-tax) price, 1-s, where $s>0$ denotes the subsidy level provided by the government, subsidization would be preferred to direct provision when the following condition holds: 
(C1) $s \cdot g(1-s) \leq g(1-s)-g(1)$

Rearranging yields:

$$
(1-s) \cdot g(1-s) \geq g(1) \leftrightarrow \int_{0}^{s}\left[-g^{\prime}(1-x) \cdot(1-x)-g(1-x)\right] d x>0
$$

Further rearranging the integral expression in $(\mathrm{C} 2)$ yields:

$$
\left|\eta_{g, 1-x}\right| \geq 1
$$

where $\left|\eta_{g, 1-x}\right|=\frac{\int_{0}^{s}\left[-g^{\prime} \cdot \frac{1-x}{g}\right] g d x}{\int_{0}^{s} g d x}$ denotes the (average) price elasticity (in absolute values) of the demand for charitable contributions over the price range $[1-s, 1]$.

Thus, when the demand for charitable contributions is sufficiently elastic (namely, the elasticity is weakly exceeding unity) subsidization would prevail over direct provision.

Two final remarks are in order. First, by continuity considerations, as providers derive utility from warm glow, the threshold elasticity above which subsidization would be preferred to direct provision would in fact fall below unity. Second, the empirical evidence on the price elasticity of the demand for charitable contributions [see Bakija (2013)] suggests that the elasticity is approximately unitary, supporting the sufficient condition given in $(\mathrm{C} 3)^{21}$

\footnotetext{
${ }^{21}$ Condition (C3) implies that subsidization would be preferred to direct provision but does not exclude the possibility of a hybrid optimum combining both direct provision and subsidization. With an iso-elastic utility from warm glow, $w(g)=g^{\gamma} / \gamma$, with $\gamma<1$, the price elasticity of the demand for charitable contributions would be given (in absolute values) by $1 /(1-\gamma)$, and condition (C3) would be equivalent to $\gamma>0$. With such a specification condition (C3) would hold for any degree of subsidization $s^{\prime}, 0<s^{\prime} \leq s$, hence the social optimum would entail no direct provision of the public good by the government.
} 


\section{Appendix D: Proof of Proposition 2}

The Lagrangean of the government optimization problem is given by:

$$
\begin{aligned}
L= & W(G, t, T)+\lambda_{1} \cdot\left[N \cdot\left[\alpha \cdot q^{h}+(1-\alpha) \cdot q^{l}\right] \cdot[g(t) \cdot(1+t)]+(N+M) \cdot T-G\right] \\
& +\lambda_{2} \cdot\left[G-N \cdot\left[\alpha \cdot q^{h}+(1-\alpha) \cdot q^{l}\right] \cdot g(t)\right],
\end{aligned}
$$

with $\lambda_{i}, i=1,2$, denoting, respectively, the Lagrange multipliers associated with the revenue constraint in (12) and the public good provision constraint in (13).

Formulating the first-order conditions with respect to $G, t$ and $T$ would yield identical expressions to (B1)-(B3):

(D1) $\frac{\partial L}{\partial G}=(N+M) \cdot h^{\prime}(G)-\lambda_{1}+\lambda_{2}=0$,

(D2) $\frac{\partial L}{\partial t}=N \cdot\left[\alpha \cdot q^{h}+(1-\alpha) \cdot q^{l}\right] \cdot\left[w^{\prime}[g(t)] \cdot g^{\prime}(t)-g^{\prime}(t) \cdot(1+t)-g(t)\right]$

$$
\begin{aligned}
& +\lambda_{1} \cdot N \cdot\left[\alpha \cdot q^{h}+(1-\alpha) \cdot q^{l}\right] \cdot\left[g^{\prime}(t) \cdot(1+t)+g(t)\right] \\
& -\lambda_{2} \cdot N \cdot\left[\alpha \cdot q^{h}+(1-\alpha) \cdot q^{l}\right] \cdot g^{\prime}(t)=0
\end{aligned}
$$

(D3) $\frac{\partial L}{\partial T}=(N+M) \cdot\left(\lambda_{1}-1\right)=0 \leftrightarrow \lambda_{1}=1$.

Substituting for $\lambda_{1}$ and $\lambda_{2}$ from (D3) and (D1) into (D2) yields upon re-arrangement:

$$
\text { (D4) } \begin{aligned}
\frac{\partial L}{\partial t}= & \underbrace{\left\{N \cdot\left[\alpha \cdot q^{h}+(1-\alpha) \cdot q^{l}\right] \cdot\left[(N+M) \cdot h^{\prime}(G)\right] \cdot g^{\prime}(t)\right\}}_{\text {Pigouvian Term }} \\
& +\underbrace{\left\{N \cdot\left[\alpha \cdot q^{h}+(1-\alpha) \cdot q^{l}\right] \cdot\left[w^{\prime}[g(t)]-1\right] \cdot g^{\prime}(t)\right\}}_{\text {Signaling Correction Term }}=0 .
\end{aligned}
$$

Notice that as $g(t) \cdot(1+t)=\bar{\theta}-\underline{\theta}$, it follows that $g^{\prime}(t)<0$; hence, condition (D4) holds if-and-only-if:

(D5) $(N+M) \cdot h^{\prime}(G)+\left[w^{\prime}[g(t)]-1\right]=0$.

As in the previous case with no signaling, one can show that the optimal solution involves no direct provision of the public good by the government. To prove this it 
suffices to show that the constraint in (13) is binding. To see this, suppose by negation that the constraint in (13) is not binding; hence, $\lambda_{2}=0$. Notice that as $w^{\prime}(g)>0$, it follows from (D5) that $(N+M) \cdot h^{\prime}(G)-1<0$. Thus, by virtue of conditions (D1) and (D3), it follows that $\lambda_{2}>0$. We therefore obtain the desired contradiction and conclude that constraint (13) is binding. This completes the proof of part (i).

The expression given in (D4) captures two conflicting effects that determine the sign of the optimal tax on contribution. The first term is negative [recalling that $g^{\prime}(t)<0$ ] and works in the direction of granting a subsidy to contributions. This term captures the standard Pigouvian motive for subsidizing contributions. The second term is positive [recalling that $w^{\prime}[g(t)]-1<0$, by virtue of (D5)] and works in the direction of levying a tax on contributions. This term captures the corrective effect of taxing contributions on the wasteful pure-signaling donations. Whether the optimal tax is positive or negative depends on the magnitudes of the aforementioned two conflicting components, reflecting the extent of the returns on signaling, captured by the term $\bar{\theta}-\underline{\theta}$. We turn now to provide a characterization of the optimal tax on contributions as a function of the returns on signaling.

Recalling that the public good provision constraint given in (13) is binding, and employing the fact that by construction of the equilibrium with signaling it follows that $g(t) \cdot(1+t)=\bar{\theta}-\underline{\theta}$, one can re-formulate the condition in (D5) to obtain:

$$
\begin{aligned}
& K[(\bar{\theta}-\underline{\theta}), t] \equiv \\
& \quad(N+M) \cdot h^{\prime}\left(N \cdot\left[\alpha \cdot q^{h}+(1-\alpha) \cdot q^{l}\right] \cdot \frac{(\bar{\theta}-\underline{\theta})}{(1+t)}\right)+\left(w^{\prime}\left[\frac{\bar{\theta}-\underline{\theta}}{(1+t)}\right]-1\right)=0 .
\end{aligned}
$$

Recalling our assumption that both $w$ and $h$ satisfy the INADA conditions, it follows that for any value of $t$, the following two conditions hold: $\lim _{(\bar{\theta}-\underline{\theta}) \rightarrow 0} K[(\bar{\theta}-\underline{\theta}), t]>0$ and 
$\lim _{(\bar{\theta}-\underline{\theta}) \rightarrow \infty} K[(\bar{\theta}-\underline{\theta}), t]<0$. Thus by the continuity of $K$, it follows by the intermediate value theorem that for any value of $t$ there exists a solution to (D6). Furthermore, as $\frac{\partial K}{\partial(\bar{\theta}-\underline{\theta})}<0$ and $\frac{\partial K}{\partial t}>0$, by virtue of the concavity of $h$ and $w$, the solution is unique. Let the (unique) solution for the condition in (D6) be denoted by $k(t)$; namely, $K[k(t), t]=0$. As $\frac{\partial K}{\partial(\bar{\theta}-\underline{\theta})}<0$ and $\frac{\partial K}{\partial t}>0$ it follows that $k^{\prime}(t)>0$. We thus conclude that $t>0$ if-and-only-if $\bar{\theta}-\underline{\theta}>k(0)$ and furthermore that $t$ is increasing with respect to $\bar{\theta}-\underline{\theta}$.

Denoting by $t^{*}$ the optimal subsidy under the regime where no signaling takes place and by $g^{*}$ the associated level of contributions by a socially motivated provider, given by the implicit solution to $w^{\prime}(g)=1+t^{*}$, it follows by virtue of conditions (D6) and (B5) that $k\left(t^{*}\right)=g^{*} \cdot\left(1+t^{*}\right)$. Assuming that signaling takes place under the optimal policy regime, it necessarily follows by virtue of (B7) that:

(D7) $k\left(t^{*}\right)<\bar{\theta}-\underline{\theta}$.

As $k^{\prime}(t)>0$, it follows that the optimal tax on charitable contributions under the regime where signaling takes place, given by the implicit solution to $k(t)=\bar{\theta}-\underline{\theta}$, satisfies $t>t^{*}$. This completes the proof of parts (ii) and (iii).

Our final step is to verify that when the price elasticity of charitable contributions and the returns on signaling are sufficiently large, our assumption that providers set their contribution above the level that maximizes the warm-glow component in their utility is consistent with the optimal solution for the government problem. 
Fix the returns on signaling and let $t^{\prime}$ denote the optimal tax on charitable contributions with signaling in place and $g^{\prime}$ denote the implicit solution to $w^{\prime}(g)=1+t^{\prime}$. Consistency requires that the following condition should hold:

(D8) $g^{\prime} \cdot\left(1+t^{\prime}\right)<\bar{\theta}-\underline{\theta}$.

Namely, socially motivated providers setting their contribution at the level that maximizes the warm-glow component in the utility function (given the optimal tax, $t^{\prime}$ ) cannot deter mimicking by non-socially motivated providers.

As the expression on the left-hand side of (D7) is independent of $\bar{\theta}-\underline{\theta}$, it follows that when the returns on signaling, as captured by the term $\bar{\theta}-\underline{\theta}$, are sufficiently large condition (D7) is satisfied. Furthermore, by virtue of part (iii) of the proposition (proved above), it follows that $t^{\prime}>t^{*}$. Condition (D7) then implies condition (D8) if the price elasticity of charitable contributions is sufficiently large. ${ }^{22}$ Thus, in line with the presumption in the statement of the proposition, when the price elasticity of charitable contributions is sufficiently large, the separating equilibrium entails signaling by socially motivated providers. This concludes the proof.

\footnotetext{
${ }^{22}$ A sufficient condition for condition (D8) to hold is that the price elasticity of charitable contributions is unitary, consistent with the existing empirical evidence [see Bakija (2013)].
} 


\section{References}

Andreoni, James. 1989, "Giving with Impure Altruism: Applications to Charity and Ricardian Equivalence", Journal of Political Economy, volume 97(6), pp. 1447-1458.

------ 1990, "Impure Altruism and Donations to Public Goods: A Theory of Warm-Glow Giving", The Economic Journal, volume 100(401), pp. 464-477.

Bakija, Jon. 2013, "Tax Policy and Philanthropy: A Primer on the Empirical Evidence for the United State and Its Implications,” Social Research 80:557-584.

Benabou, Roland and Tirole, Jean. 2010, "Individual and Corporate Social Responsibility," Economica, 77:1-19.

Blumkin, Tomer and Sadka, Efraim. 2007, "A case for taxing charitable donations", Journal of Public Economics, volume 91, pp. 1555-1564.

Cho, In-Koo and Kreps, David M. 1987, "Signaling Games and Stable Equilibria," The Quarterly Journal of Economics, 102: 179-222.

Elfenbein, Daniel, Fisman, Ray, and Mcmanus Brian. 2012, "Charity as a Substitute for Reputation: Evidence from an Online Marketplace," Review of Economic Studies 79: $1441-1468$.

Glazer, Amihai and Konrad, Kai. 1996, "A Signaling Explanation for Charity," American Economic Review, vol. 86(4), pages 1019-1028.

Levmore, Saul. 2008, “Taxes as Ballots” 65 U. CHi. L. REV. 387.

Mayer, Lloyd Hitoshi and Ganahl, Joseph R. 2014, "Taxing Social Enterprise," 66 Stanford Law Review 387.

Siegel, Donald S. and Vitaliano, Donald F. 2006, "An Empirical Analysis of the Strategic Use of Corporate Social Responsibility," Journal of Economics and Management Strategy, volume 16(3), pp. 773-792. 
Figure 1: The Optimal Tax as a Function of the Returns on Signaling

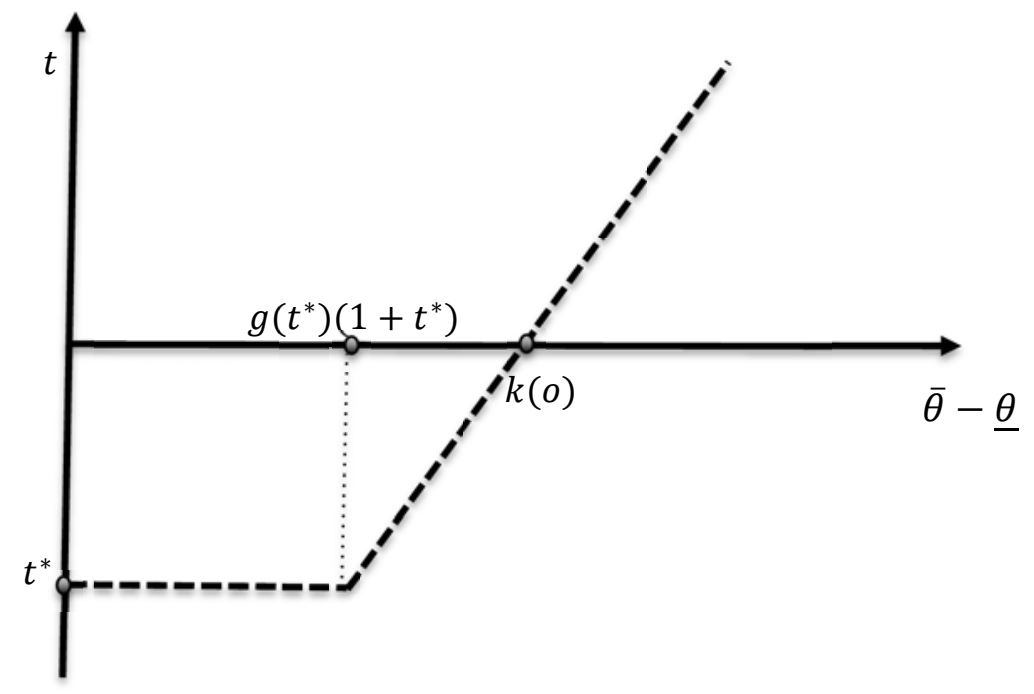


Figure 2: The Effective Marginal Tax as a Function of the Returns on Signaling

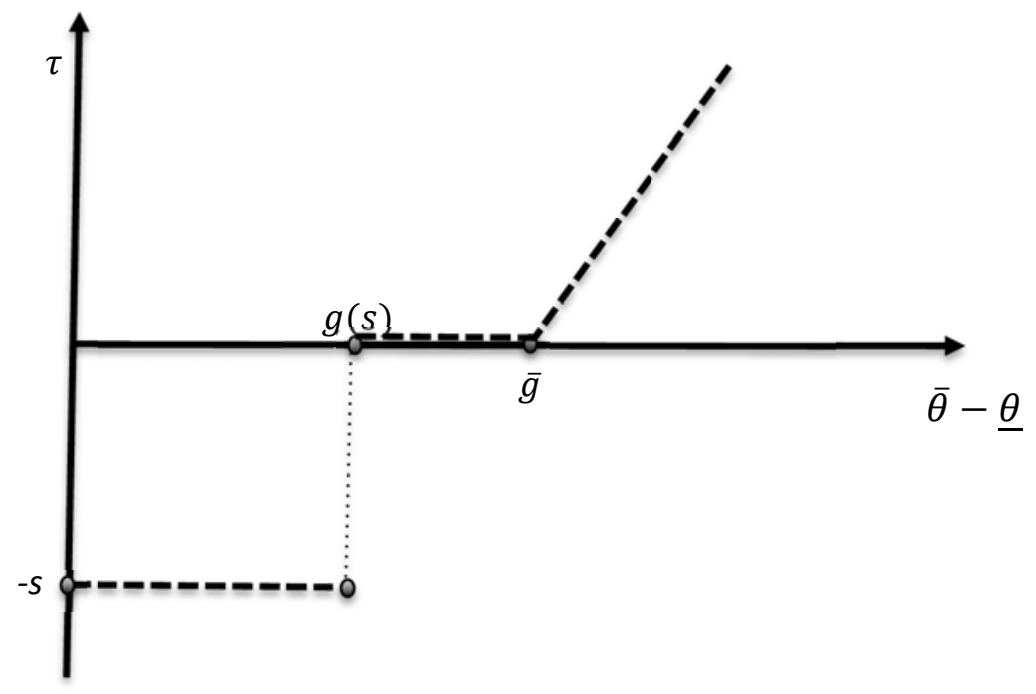

